\title{
Mass Spectrometry of Biomolecules: From PDMS to MALDI
}

\author{
Ronald D. Macfarlane \\ Department of Chemistry \\ Texas A \&S M University \\ College Station, Texas, USA
}

May, 1999

\begin{abstract}
Mother Nature (the Ultimate Chemist): South America holds some of the richest rain forests in the world where Nature, through the abundance of the essentials of life has produced a rich spectrum of biological molecules. The early inhabitants of these regions, over thousands of years, have, through experimentation, identified plants and animals that contain components that they have found to be beneficial for their health or so toxic that they can be used as a weapon.
\end{abstract}

Do any of you know any examples of drugs or toxins that have evolved to commercial products that have had their origin from the experience of pre-modern history South Americans?

While the knowledge of these important biological compounds was passed on through generations from stories and the practice of medicine by learned members of these native communities, until recently, these stories and "home remedies" were not recognized by the science community as having much validity. Long before penicillin was discovered, the Native people knew that a bad wound would heal more quickly if moldy bread were layered over the wound.

Although we have reached a high level of sophistication in synthesizing biological compounds, Nature, through the experience of millions of years, has developed synthesis pathways that are marvels in their complexity and ingenuity. Consequently, many scientists are not only isolating and identifying the active ingredients of a plant but also elucidating the pathways that Nature uses to produce that species. The countries of South America have been a rich natural laboratory for these activities.

\section{Poisonous South American tree frogs}

and mass spectrometry

When a plant or animal is identified as having pan of its chemical make-up, a species that has important physiological properties, that species is first isolated and purified. The 3-dimensional chemical structure is elucidated utilizing a variety of spectroscopic tools. The most information rich of these methods are NMR, IR, and x-ray diffraction of pieces of the molecule and the whole molecule. A structure is proposed that fits all of this information and a molecular mass determination is made that is a direct measure of the inventory of the atoms comprising the molecule. If the measured mass does not fit the proposed structure, them something is wrong with the proposed structure and it must be modified. The molecular mass determination is made by converting molecules of the species into gas phase molecular ions and the mass is measured by the interaction of these ions with magnetic or electric fields or a combination of these.

The specific example that illustrates this scenario is given by the Panamanian tree frog whose skin contains a highly toxic molecule called tetrodotoxin. It's proposed structure based on spectroscopic evidence is given below: 

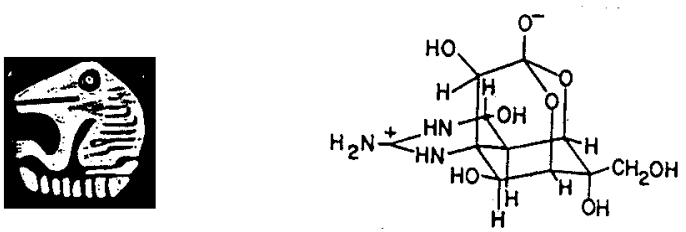

Figure 1. Proposed Structure of Tetrodotoxin.

This molecule interferes with the electrical circuitry of the heart and induces cardiac arrest. It is used in heart research to induced heart attacks in laboratory animals and to study the physiological action of this event. Attempts to obtain the molecular mass of this molecule by mass spectrometry in the early 1970 's failed because no gas phase molecular ions could be produced in the mass spectrometer. In fact, a large fraction of Nature's biomolecules could not be studied by mass spectrometry in that era because of this fundamental problem. The reason for this can be understood if the tetrodotoxin structure is examined in more detail. The highly polar groups on the structure result in a very strong dipole/dipole interaction between neighboring molecules in a sample of tetrodotoxin which means that it is difficult to pull them apart by thermal agitation. When the sample is heated to increase the intermolecular excitation, the thermal energy excites internal vibrations of the molecule and it dissociates. This process is depicted below.
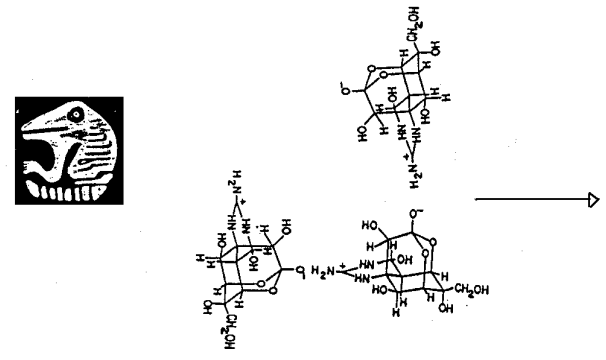

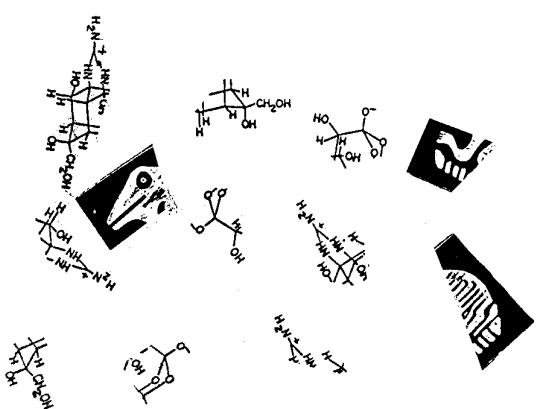

Figure 2. Thermal excitation of a tetrodotoxin matrix leading to decomposition.

\section{A Chance Observation in a Nuclear Physics Study Leads to a Solution to the Problem}

In 1972, our group at Texas A\&M University was studying the emission of radioactive recoil ions from surfaces using time-of-flight mass spectrometry. In addition to the expected ions, molecular ions of impurities on the surface also appeared in the mass spectrum which were postulated to be due to the ionization of molecules on the surface of the sample by the nuclear radiation. To test this hypothesis, a ${ }^{252} \mathrm{Cf}$-source was placed behind the sample and fission fragments from the spontaneous of this nuclide irradiated the sample. Molecular ions of surface molecules were indeed produced in high abundance and detected in the mass spectrum verifying the origin of the molecular ions in the nuclear physics experiment. Key individuals participating in this study in 1972 in our lab are participating in the Desorption '98 meeting this week. They are
Karl Wien and Hartmut Jungclas from Germany. This phenomenon was so unexpected and interesting that it became the topic of our future studies and the nuclear physics interests gradually dwindled to obscurity. We began to coat the surface with a variety of different materials including those with biological significance and in all cases, we obtained molecular ions of whatever was put on the surface. My nuclear physics colleagues who were working on problems in the same nuclear physics field also became interested in this new adventure, and since they all had the same nuclear physics technology were able to do the same thing in their lab. Many of these individuals, (nuclear physicists turned mass spectroscopists) are also participating in this week's activities and have made major contributions to the field over the past 2 decades. In addition to Wien and Jungclas, they include Yvon Le Beyec, Bo Sundqvist, Ken Standing, Helmut Voit, and Brian Chait. 


\section{Back to the Poisonous Frog and the Molecular Decomposition Problem}

In 1973-4, when our studies on the use of fission fragments of ${ }^{252} \mathrm{Cf}$ to generate mass spectra was beginning to evolve, one of the students in my group began his graduate studies at Stanford University working for Harry Mosher, a renowned natural products chemist. Professor Mosher hid been studying the tetrodotoxin structure problem for several years and proposed the structure shown above. He was not able to verify this structure by MW determination because of the involatility/decomposition problem. He had tried for several years using every new mass spectrometric method that had evolved but with no success. At the urging of his new student, he sent us a sample to run and we got a good result. At that time, we didn't know it was a tough problem and we also didn't know about the problem mass spectrometry was having with involatile biomolecules. The spectrum we got by shooting fission fragments through a sample of this toxin is shown below.
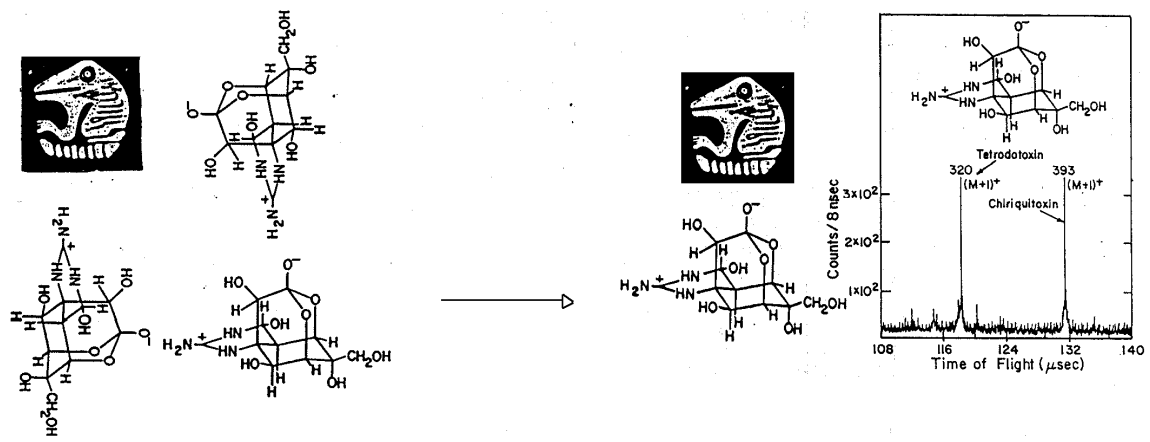

Figure 3. First Mass Spectrum of tetrodotoxin obtained by PDMS

\section{Naming the Method}

Professor Mosher was very excited about this result. He suggested that we forget about this nuclear physics stuff and really develop the method into a technology for natural product studies. He also insisted that we come up with a name for it, something organic chemists could pronounce and remember and, after several attempts, came up with ${ }^{252} \mathrm{Cf}$ PDMS or PDMS (Plasma Desorption Mass Spectrometry).

\section{So how do Fission Fragments solve the Problem?}

A fission fragment is a high energy, highly-charged ion that is a highly destructive species. The surprise was that a component of its interaction with solids is gentle, resulting in the desorption and ionization of fragile molecules without decomposing them. Later studies showed that the combination of high energy density and short-excitation time was they key feature of the interaction that makes PDMS work and that even very large biomolecules can survive the hostile environment of the fission track and become intact gas phase molecular ions.

\section{PDMS Research, 1974-1984}

During this era, our laboratory at Texas A\&M was flooded with requests to "run samples", primarily to determine the MW of molecules of biological importance. For the most part, these were pharmaceuticals being developed by drug companies and the MW determination was a requirement for acceptance as a drug for the public. Many of these compounds were known only by a code. We got some nice looking spectra but had no idea what it all meant. We must have been quite successful because the sample came flooding in. This was a confusing time for us because we were still more nuclear scientist than mass spectroscopist, and in fact, we knew very little about the field. We now know that many of the samples we studied have become important drugs currently in use. These include vancomycin, bleomycin, 
amphotericin, and thiostreptin. Although we were successfull with these molecules, the mass spectrometry community did not embrace our endeavors for several reasons: fear of radioactivity, use of time-of-flight and not a magnet or quadrupole, low mass resolution, and you had to wait for more than a minute to obtain the mass spectrum. It was a time when editors of journals were more sympathetic to our manuscripts than reviewers.

\section{Accelerator Studies, 1976-present}

During the time when we were actively involved in "running samples", my nuclear physics colleagues, who, like most physicists, generally distain chemistry, began to tackle the problem of mechanism of PDMS using their nuclear accelerators. It was from these experiments, that the properties of the incident ion were elucidated and the interaction of high energy ions with solids as a field in its own began to emerge. These activities were initiated and sustained by Voit at Erlangen, Sundqvist at Uppsala, Le Beyec at Orsay, Wien at Darmstadt, da Silveira at Rio and Tombrello at Caltech. Intense and enduring friendships with these groups for the past 25 years have been one of the richest components of my professional and private life. We have maintained this closeness through the series of Desorption ' $\mathrm{xx}$ meetings that have taken place in wonderful and interesting sites that include Brazil as a twotime favorite of this group. Over the years, the group has expanded as the field has grown and developed new roots and branches. It is this feature that I wish to address next.

\section{Secondary Ion Mass Spectrometry (SIMS)}

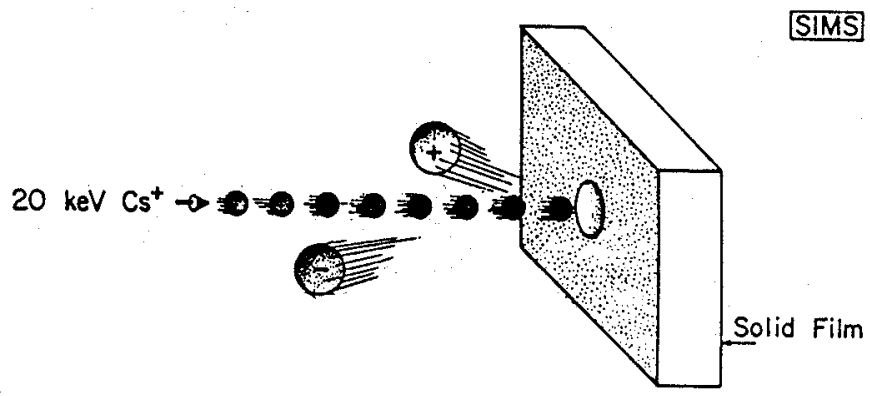

This method was well established before PDMS came along. Low energy ions bombard the surface of a sample producing gas phase ions of the matrix. The method was used extensively for characterizing the surfaces of inorganic species before 1974 and continues today. The mechanism involves collisions of the incident ion with surface atoms that are ejected. Molecular ions were also present in the mass spectrum which were an "unwanted background". When PDMS was introduced, it was realized that a component of the SIMS process also was similar to PDMS, the rapid deposition of energy, and molecular ions of involatile molecules could also be desorbed by these low energy ions. From 1976 to the present, SIMS has been a member of the Desorption ' $\mathrm{xx}$ family, led by Benninghoven at Munster and Standing in Winnipeg. A friendly rivalry emerged during this era where a series of papers were presented on SIMS catching up to PDMS in terms of number of ions desorbed and the mass range. In terms of acceptance by the mass spec. community, SIMS had the same problem as PDMS because it did not use sophisticated magnets and have high mass resolution. This acceptance issue comes up in several places here because it was a real dilemma. Thousands of mass spec. people were essentially ignoring the work of the PDMS/SIMS people despite the successes.

\section{The Beginning of Being Accepted}

The first mass spectrometry meeting where PDMS results were presented was in 1976 . It was the last paper of a week-long meeting and 4 people were in the audience (they were waiting for their plane). But as it turned out, they were very important people and each became part of the PDMS family. They were highly respected in the mass spec. community politics and 3 of the 4 became President of the American Society for Mass Spectrometry and the fourth is now President of a large mass spectrometry company in the US. It was through these individuals and their foresight and unselfish devotion to the good of the field that PDMS began to be a part of this community. To Frank Field, Hank Fales, Jim McCloskey, and Ian Jardine, the Desorption ' $\mathrm{xx}$ group can be grateful to these bridge builders. For Frank Field and Victor Tal'Rose, their strong intellectual leadership became the base for acceptance by the mass spectrometry community. 


\section{The Competition Heats Up, Laser Desorption is Introduced}

It was generally accepted by the mass spectrometry community that there must be a better way than PDMS to obtain mass spectra of biomolecules but using the same general idea. In the late 1970's, Meuzzelar in Holland began to obtain impressive mass spectra using laser excitation. When I heard one of his first talks on his work, I had the feeling that the days of the monopoly that PDMS had on the field were numbered. Laser desorption had so much going for it in terms of control of the excitation. The pulsed laser was a natural for the TOF technique. However, the promise did not convert to reality in the next few years. One of the Desorption 'xx participants, Franz Hillenkamp, took up the challenge of laser desorption at that time and for several meeting Hillenkamp kept us informed on the progress of the development of the method. The mass spectrometry community and commercial mass spectrometer makers patiently waited for something more attractive.

\section{Fast Atom Bombardment (FAB) makes its debut}

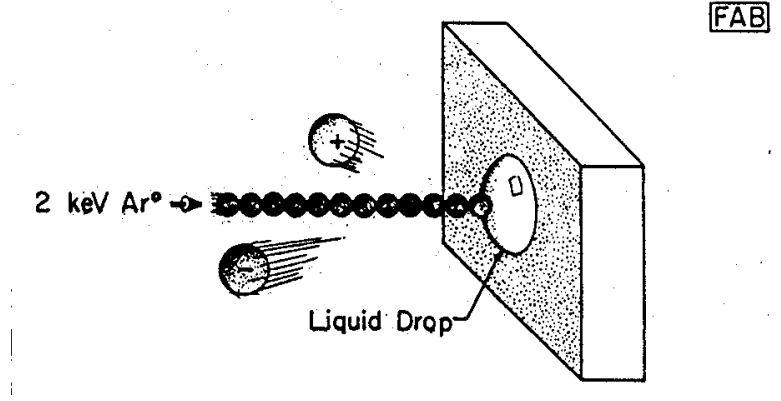

In the early 1980's, we were working with Kenneth Reinhart, a natural product chemist at Illinois, on a series of potentially important antibiotics derived from marine organisms. These were tough molecules to analyze. In the middle of the studies, Ken told us about a method he heard was just developed in England that was an important breakthrough for the analysis of involatile biomolecules. He was going to England immediately with his samples and would report back. When he returned, he told the story of a clandestine operation literally clocked in secrecy where he handed his samples to an operator who then went behind a curtain, prepared the samples and inserted them into a mass spectrometer. Out came high quality spectra and the big news was that a "proper" mass spectrometer was used i. e. a magnetic sector instrument. The professor in charge was Michael Barber and the method is known as fast atom bombardment. The incident species is a high energy neutral atom and the matrix is a liquid matrix. A high yield of ions, a steady current of these ions is produced, and it is compatible with the technology currently in use by the mass spectrometry community at that time. High resolution, instant gratification in the time required to get the data, important criteria for acceptance, was a part of the FAB technique. Within the next year, I heard Mickey Barber present his method and results at a meeting in London, the last talk of the meeting, and I knew then that PDMS was on its way to become part of the past history of mass spectrometry. Most of the important pharmaceutical problems could now be solved by FAB and the demand for PDMS measurements was reduced to almost nothing. The mass spectrometry community embraced FAB and there was a public impression that now there was a method far analyzing involatile biomolecules. This perception was not challenged by the Desorption 'xx community (I'm not sure why; intellectual maturity?, unselfish devotion to the science and not the publicity?, .....?) and the FAB researchers chose not to be a part of the Desorption 'xx community even though the mechanisms of the process were closely related. In later years, Mickey and I had several good interactions and conversations. In the last (I didn't know it would be the last), I got up the courage to ask him a question I had pondered since I first heard about FAB and the basis for the question was the lack of the development of a link between FAB and the other desorption/ionization methods. My question: "Mickey, did PDMS have any influence on the development of FAB? Mickey: "You damn right it did! We were so angry that you were able to run all those samples on a TOF instrument that we put one of your spectra on the wall of our lab as a daily reminder that we must find a way to run these samples on a proper mass spectrometer." I cherish that moment. 


\section{The Post-FAB era and the birth of MALDI}

What kept PDMS going after FAB was introduced was the ability to study larger biomolecules. Largely through the work of the Uppsala group, the mass range limit was pushed up to close to 40,000 for a protein. But it was hard work. At the same time, biotechnology and medicine was becoming more sophisticated and the desire to obtain accurate and precise molecular weights of biopolyimers was becoming more acute. It was obvious from our studies that there was a limit to the size of a protein that could be desorbed by a fission fragment. This was a bit of disappointment because our own interests were heading in a direction in medical research where we wanted to use PDMS to characterize proteins involved in human health and disease. It was clear that PDMS was not going to be used in these studies. In the late 1980's, at an International Science meeting in Bordeaux, Franz Hillenkamp gave a talk, imbedded deep in the program on the work he and Michael Karas were doing with laser desorption; a breakthrough and a new acronym: MALDI. You will hear more about this method from Franz in the next day or two. This time, my response to his talk was absolute excitement! I could see my desire to have a method available to accomplish my long term goal: to make a contribution to medicine, had a chance. I close this long set of lecture notes with an excerpt of dream that has become reality.

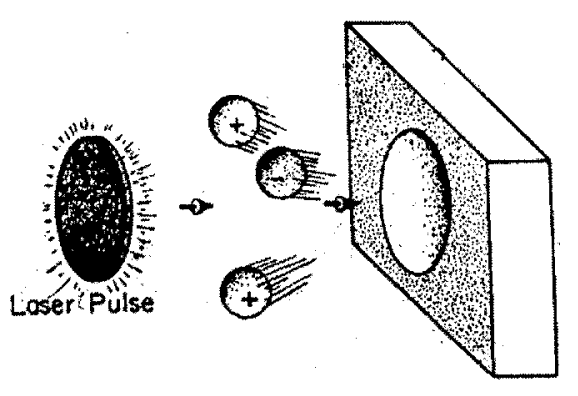

Dateline: January 30, 1998, Scott \& White Hospital, Temple, Texas. A 51 year old white female admitted to emergency room with chest pain rated as 9 out of 10 with radiation to the upper jaw and associated with shortness of breath, nausea, and diaphoresis. A new technique for monitoring the severity of the heart damage is employed. MALDI spectra, recorded over a period of 3 days on the proteins present in the patients HDL, shows an increase in the concentration of the acute phase response protein, serum amyloid SAA, an indication of extensive damage to the inferior myocardial wall. Attending physician, Catherine J. McNeal, MD, Ph.D. 


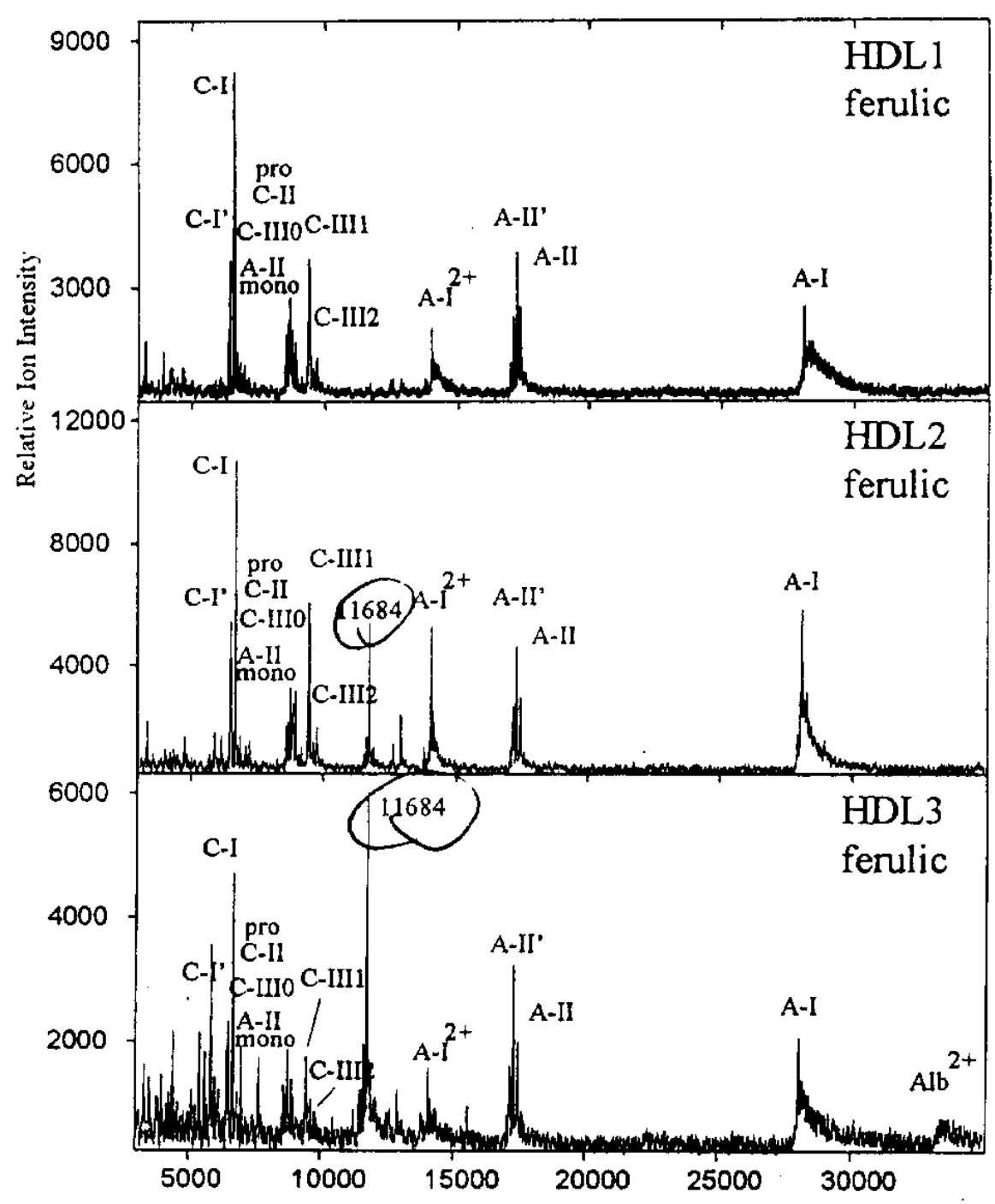

MALDI spectra of a heart patient recorded over a period of 3 days. (A special thanks to Dave Russell and his group in the Texas A\&M, Department of Chemistry, Laboratory for Biological Mass Spectrometry for obtaining these data for us. It was carried out on a very long Perspective Biosystems Instrument). 